\title{
Liver Sarcoidosis That Presented with Dysphagia
}

\author{
Miharu Samuraki ${ }^{1}$, Takuya Nakahashi ${ }^{1}$, Shou Aoyama ${ }^{1}$, Yoshinobu Hinoue ${ }^{1}$, \\ Hirokazu Takahashi ${ }^{1}$, Katsuhiko Saito ${ }^{2}$ and Shigeru Hayashi ${ }^{1}$
}

\begin{abstract}
We report a case of liver sarcoidosis with dysphagia. Although akinesia of the soft palate, disappearance of the palatal reflex, and pyramidal signs indicated brainstem lesions, brain magnetic resonance imaging showed no lesion and the focus was obscure. Iritis, parotiditis, and hilar lymphadenopathy supported the diagnosis of sarcoidosis. However, lung biopsy was normal. Finally, sarcoidosis was diagnosed by liver biopsy. We speculated that microgranulomas in the brainstem that did not exhibit imaging abnormalities causing the neurological symptoms and that liver biopsy can be an effective diagnostic tool even for cases that presented with neurological signs, but no gastrointestinal symptom.
\end{abstract}

Key words: sarcoidosis, dysphagia, liver biopsy

(Inter Med 47: 1259-1261, 2008)

(DOI: 10.2169/internalmedicine.47.1050)

\section{Introduction}

Sarcoidosis is a granulomatous inflammatory disease involving multiple organ systems. Dysphagia is a common symptom in patients with sarcoidosis, and there have been many mechanisms to explain the presence of the symptom, such as esophageal involvement (1-4), laryngeal involvement (5), cranial neuropathy (6-10), and brainstem granuloma (11). In particular, cranial neuropathy in patients with sarcoidosis frequently causes dysphagia (12). Meanwhile, only one case has been reported of sarcoidosis that presented with dysphagia and revealed histologically brainstem microgranuloma without magnetic resonance imaging (MRI) abnormalities (13). Additionally, to our knowledge, there have been no reports of liver sarcoidosis that presented with neurological symptoms as the first symptom, and with no gastrointestinal symptom. Herein, we report a case of liver sarcoidosis that presented with neurological symptoms and was indicated to have brainstem involvement despite the lack of abnormalities on MRI, and was finally diagnosed by liver biopsy.

\section{Case Report}

A 34-year-old woman presented with a one-month history of bilateral dysesthesia of the hands, a two-week history of increasing dysphagia, and nasal voice. She was admitted to our hospital, because laryngoscopic examination showed salivary retention at the hypopharynx. She was anemic and had taken medication since the age of 29 years. She also had arteriovenous malformation in the right occipital lobe of the brain that was diagnosed at 29 years of age. Since she did not exhibit any symptoms, she did not undergo an operation at that time. She had no significant family history.

On admission, she was afebrile and her blood pressure was $140 / 90 \mathrm{mmHg}$. She exhibited bilateral swelling of the parotid glands with mild tenderness and a $4 \mathrm{~cm}$ soft nodule of the neck without tenderness. She exhibited no skin lesion. Her consciousness was alert. Meningeal signs were absent. Bilateral soft palate showed no elevation, and the gag and palatal reflexes had disappeared. She presented with dysarthria due to severe nasal voice and she could not swallow anything at all. The remaining functions of the cranial nervous system were normal. There was no weakness or atrophy in the sternocleidomastoideus or trapezius. She had weakness in the neck, but not in her limbs. Deep tendon reflexes were brisk and pathological bilateral reflexes were elicited in the legs. She felt dysesthesia in her fingers, but there were no objective sensory abnormalities. She presented with no ataxia, gait disturbance or autonomic disorder. Akinesia of the soft palate, disappearance of the gag and palatal

${ }^{1}$ Department of Internal Medicine, Toyama City Hospital, Toyama and ${ }^{2}$ Department of Pathology, Toyama City Hospital, Toyama Received for publication February 26, 2008; Accepted for publication April 1, 2008

Correspondence to Dr. Miharu Samuraki, kimiharu@beige.plala.or.jp 
reflexes and pyramidal signs indicated that her symptoms were due to a brainstem lesion.

Urinalysis showed no abnormality. Blood cell counts indicated microcytic anemia ( $\mathrm{Hb} 9.3 \mathrm{~g} / \mathrm{dL}$ ). Routine blood analysis showed that the remaining hemogram, coagulation, liver function, and renal function were within normal limits. AST was $13 \mathrm{IU} / \mathrm{L}$, ALT $9 \mathrm{IU} / \mathrm{L}, \gamma$-GTP $18 \mathrm{IU} / \mathrm{L}$, alkaline phosphatase $141 \mathrm{IU} / \mathrm{L}$, LDH $140 \mathrm{IU} / \mathrm{L}$, total bilirubin 0.7 $\mathrm{mg} / \mathrm{dL}$. Immunoglobulin $\mathrm{G}$ (IgG) was $1,387 \mathrm{mg} / \mathrm{dL}$, angiotensin-converting enzyme (ACE) $12.8 \mathrm{U} / \mathrm{L}$ (normal: 7.0-25.0 U/L). Lysozyme and $\beta_{2}$-microglobulin were also within normal limits. Soluble interleukin-2 receptor was slightly elevated to $898 \mathrm{U} / \mathrm{mL}$ (normal: 190-650 U/mL). Antinuclear antibody, antineurophil cytoplasmic antibody, antiacetylcholine receptor antibody, and the other antibodies were negative. CSF showed elevation of cell counts $[9 / \mu \mathrm{L}$ (normal: $0-5 / \mu \mathrm{L}$ )] and proteins [57 mg/dL (normal: 15-45)], but normal sugar and IgG index. No malignant cells were observed.

The chest X-ray and subsequent computed tomography (CT) showed right hilar lymphadenopathy. Electrocardiogram and echocardiography were normal. Brain and cervical MRI with gadolinium contrast did not reveal abnormal findings, except for the arteriovenous malformation in the right occipital lobe. CT scan of the pharynx indicated no lesion resulting in external compression of pharynx. ${ }^{67} \mathrm{Ga}$ scintigraphy revealed right hilar and bilateral parotid gland uptake. Laryngoscopic examination did not indicate hypomobility of vocal cords, but did show salivary retention at the hypopharynx. Upper endoscopy was macroscopically normal. Slit lamp examination revealed bilateral iritis. Ultrasound of the abdomen showed no abnormalities. Tuberculin test was positive. Bronchoscopy and transbronchial biopsy did not show any alterations. Bronchoalveolar lavage obtained $4 \%$ neutrophils, $21 \%$ lymphocytes, and $75 \%$ macrophages. T cells accounted for $96.6 \%$ and B cells less than $1 \%$ of all lymphocytes. However, unfortunately, the CD4/8 ratio could not be measured due to some technical problems. Tuberculosis polymerase chain reaction (PCR) was negative. Nerve conduction studies showed normal findings. Repetitive nerve stimulation demonstrated no reduction in responses. Intravenous injection of edrophonium chloride did not improve her symptoms.

Iritis, swelling of the parotid glands, and hilar lymphadenopathy indicated that she was suffering from sarcoidosis. However, there was no evidence of sarcoidosis on transbronchial biopsy. Moreover, we could not perform biopsy of the nodule of the neck because the nodule had immediately vanished. Therefore, we performed liver biopsy to confirm the diagnosis, based on the report that liver granulomas can be found in $60-90 \%$ of systemic sarcoidosis patients with involvement of the gastrointestinal tract $(14,15)$. The liver needle biopsy showed two granulomatous foci at low magnification, which at higher magnification were found to be noncaseating and contained multiple giant cells (Fig. 1).

Based on the results of the liver biopsy, she was diag-

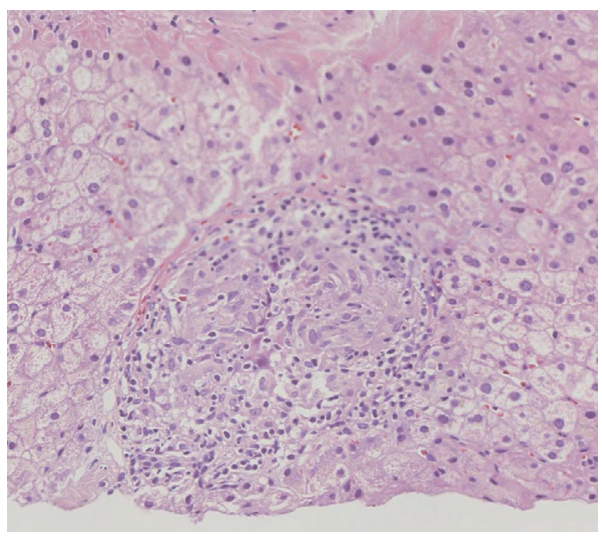

Figure 1. Microscopic findings in the liver with a noncaseating granuloma composed of giant cells. Hematoxylin and Eosin staining $(\times 400)$.

nosed as liver sarcoidosis with neurological signs (16). Initial treatment with two courses of corticosteroid pulse therapy was immediately effective. She was able to eat and speak gradually, and did not require additional total parenteral nutrition after the first pulse therapy. After beginning continuous oral prednisolone $60 \mathrm{mg} /$ day, dysesthesia and weakness of the neck also improved. While undergoing corticosteroid therapy, she suffered from steroid myopathy. We gradually decreased the prednisolone to $35 \mathrm{mg} /$ day and thereafter, the steroid myopathy improved with no exacerbation of the symptoms of sarcoidosis.

\section{Discussion}

Many causes of dysphasia in sarcoidosis have been reported, as follows: (1) esophageal involvement (1-4), (2) compression of the esophagus from lymphadenopathy (17, 18), or from infiltration of Auerbach's plexus (19), (3) laryngeal involvement (5), (4) myopathy $(20,21)$, (5) neuropathy (6-10), and (6) granuloma of the skull base (22) or brain parenchymal mass (11). For this case, esophageal involvement, compression of the esophagus and laryngeal involvement were negative on pharynx CT, laryngoscopy and upper endoscopy. Dysphasia, dysarthria, pyramidal signs and disappearance of the gag and palatal reflexes indicated involvement of both of cranial nerves and corticospinal tracts. And, there is also a possibility that brainstem lesion extending from the medulla oblongata to the cervical spinal cord caused all of these symptoms. However, imaging examination, including MRI, found no lesion in the central nervous system. Although, the elevation of cell counts and proteins in the CSF indicated intramedullary or leptomeningeal lesion, the true lesions in our case were obscure. Nishie et al described a case of sarcoidosis that was similar to our case (13). Their case presented with dysarthria, dysphasia and weakness of the upper and lower extremities. Although no abnormalities were noted with brain MRI, histologically they observed brainstem parenchymal lesions consisting of many microgranulomas. The present case could also have a 
possibility to have microgranulomas of the brainstem that do not exhibit MRI abnormalities, as with their case, but it has not been verified yet.

In regard to liver biopsy, liver granulomas can be found in $60-90 \%$ of patients with systemic sarcoidosis involving the gastrointestinal tract $(14,15)$. Since granulomas in the liver are small and predominantly located in the portal space (14), hepatic involvement is asymptomatic and associated with no abnormal liver function or mild derangement in most patients with sarcoidosis $(14,23)$. Based on these facts, we considered that it was probable that granulomas could be detected in the liver despite normal liver function, and we therefore performed liver biopsy. Liver biopsy is considered to be an effective diagnostic tool when sarcoidosis is suspected, but it is not definite.

\section{References}

1. Geissinger BW, Sharkey MF, Criss DG, Wu WC. Reversible esophageal motility disorder in a patient with sarcoidosis. Am J Gastroenterol 91: 1423-1426, 1996.

2. Hardy WE, Tulgan H, Haidak G, Budnitz J. Sarcoidosis. a case presenting with dysphagia and dysphonia. Ann Intern Med 66: 353-357, 1967.

3. Kerley P. Sarcoidosis Modern Trends in Diagnostic Radiology. In: McLaren JW, Ed. Harper \& Row, New York, 1948.

4. Wiesner PJ, Kleinman MS, Condemi JJ, Resnicoff SA, Schwartz SI. Sarcoidosis of the esophagus. Am J Dig Dis 16: 943-951, 1971.

5. Bower JS, Belen JE, Weg JG, Dantzker DR. Manifestations and treatment of laryngeal sarcoidosis. Am Rev Respir Dis 122: 325$332,1980$.

6. Castroagudin JF, Gonzalez-Quintela A, Moldes J, Forteza J, Barrio E. Acute reversible dysphagia and dysphonia as initial manifestations of sarcoidosis. Hepatogastroenterology 46: 2414-2418, 1999.

7. Garrod O. Sarcoidosis with dysphagia, peripheral neuropathy and multiple cranial nerve lesions. Proc R Soc Med 57: 175, 1964.

8. Ogawa Y, Higuchi E, Koga H, et al. [A case of sarcoidosis presenting with hoarseness and dysphagia due to glossopharyngeal and vagus nerve paresis]. Nihon Kyobu Shikkan Gakkai Zasshi 32: 602-605, 1994 (in Japanese, Abstract in English).

9. Singh MD, Fitzpatrick MJ. Cranial neuropathy associated with sarcoidosis. Case report. Dis Chest 45: 431-435, 1964.

10. Zuniga G, Ropper AH, Frank J. Sarcoid peripheral neuropathy. Neurology 41: 1558-1561, 1991.

11. Christoforidis GA, Spickler EM, Recio MV, Mehta BM. MR of CNS sarcoidosis: correlation of imaging features to clinical symptoms and response to treatment. AJNR Am J Neuroradiol 20: 655669, 1999.

12. Sakuta M. Neurosarcoidosis. Nippon Rinsho 52: 1590-1594, 1994 (in Japanese, Abstract in English).

13. Nishie M, Mori F, Suzuki C, et al. Disseminated intraparenchymal microgranulomas in the brainstem in central nervous system sarcoidosis. Neuropathology 25: 361-364, 2005.

14. Harder H, Buchler MW, Frohlich B, et al. Extrapulmonary sarcoidosis of liver and pancreas: a case report and review of literature. World J Gastroenterol 13: 2504-2509, 2007.

15. Sharma OP. Sarcoidosis. clinical, laboratory, and immunologic aspects. Semin Roentgenol 20: 340-355, 1985.

16. Sakuta M, Kumamoto $T$, Iizuka $T$, et al. Diagnostic criteria of neurosarcoidosis. Brain Nerve 58: 471-476, 2006 (in Japanese, Abstract in English).

17. Cappell MS. Endoscopic, radiographic, and manometric findings in dysphagia associated with sarcoid due to extrinsic esophageal compression from subcarinal lymphadenopathy. Am J Gastroenterol 90: 489-492, 1995.

18. Cook DM, Dines DE, Dycus DS. Sarcoidosis. report of a case presenting as dysphagia. Chest 57: 84-86, 1970.

19. Dufresne CR, Jeyasingham K, Baker RR. Achalasia of the cardia associated with pulmonary sarcoidosis. Surgery 94: 32-35, 1983.

20. Nidiry JJ, Mines S, Hackney R, Nabhani H. Sarcoidosis. a unique presentation of dysphagia, myopathy, and photophobia. Am J Gastroenterol 86: 1679-1682, 1991.

21. Takuma H, Murayama S, Watanabe M, Saito Y, Ugawa Y, Kanazawa I. A severe case of subacute sarcoid myositis. J Neurol Sci 175: 140-144, 2000.

22. Vasan NR, Allison RS. Sarcoidosis presenting as hoarseness and dysphagia. Aust N Z J Surg 69: 751-753, 1999.

23. Mueller S, Boehme MW, Hofmann WJ, Stremmel W. Extrapulmonary sarcoidosis primarily diagnosed in the liver. Scand J Gastroenterol 35: 1003-1008, 2000.

(C) 2008 The Japanese Society of Internal Medicine http://www.naika.or.jp/imindex.html 